\title{
Topical Flurbiprofen: an Effective Treatment for Episcleritis?
}

A randomised double-blind trial comparing Gutt. prednisolone $0.3 \%$ Gutt. flurbiprofen $0.03 \%$ and Gutt. saline $0.5 \%$ in the treatment of episcleritis.

\author{
C. J. LYONS, K. N. HAKIN, P. G. WATSON
}

London

\begin{abstract}
Summary
Topical flurbiprofen was compared with placebo and prednisolone $0.3 \%$ in the treatment of episcleritis by a randomised double-blind trial. Seventy-seven eyes were included in the trial. Rapid spontaneous improvement in symptoms and signs was noted in the majority of cases.

There was no significant difference between the cure-rates of patients treated with flurbiprofen and placebo over a 3-week trial period. The proportion of patients treated with prednisolone $0.3 \%$ who were cured was significantly higher than in either of the other groups after 3 weeks.
\end{abstract}

Episcleritis is generally considered to be a benign condition. Although patients complain of discomfort and of the appearance of their inflamed eye, the problem is recognised as self-limiting. However, it represents one end of the spectrum of ocular inflammatory disease, with necrotising scleral disease at the other. Previous authors ${ }^{1}$ have suggested that resolution of the inflammatory episode can be hastened by topical treatment with betamethasone or, to a lesser extent by non-steroidal anti-inflammatory drugs such as Tanderil. Currently, topical steroids are the mainstay of the treatment of episcleritis; however they are known to give rise to an inflammatory rebound phenomenon as well as to other welldocumented ocular side-effects such as reactivation of Herpes Simplex Keratitis.

Flurbiprofen is one of the few non-steroidal anti-inflammatory drugs which has been found to be effective in the management of scleral inflammatory disease. ${ }^{2}$ However, like other drugs in this class, oral administration is occasionally complicated by symptoms of heartburn and by gastric or duodenal ulceration. An effective topical anti-inflammatory drug would undoubtedly be useful in the management of scleral as well as episcleral disease. Recently, flurbiprofen has bcome available in drop form, marketed for use in the prevention of surgically-induced miosis.

We assessed its use in the clinical management of episcleritis by a randomised doubleblind trial.

\section{Method}

Consecutive patients presenting with episcleritis to the Casualty department at Moorfields Eye Hospital between August 1st 1988 and April 1st 1989 were recruited to the study after informed consent had been obtained.

The age, sex and racial origin of the patients were recorded. A detailed history was taken, with particular attention as to whether this was the first or a recurrent episode, the duration of the episode, the patient's past medical

From: Moorfields Eye Hospital, City Road, London

Correspondence to: C. J. Lyons, Moorfields Eye Hospital, City Road, London EC1V 2PD. 
history, any current medication, and any allergies.

The first 30 patients in the series were assessed by two observers ( $\mathrm{CJL}, \mathrm{KH}$ ) for standardisation of scoring. Thereafter each patient was assessed by one of these observers only.

The severity of their symptoms (pain) was graded from 0 to $3(0=$ absent, $1=$ mild discomfort, $2=$ moderate pain, $3=$ severe pain).

Conjunctival and episcleral injection were each assessed according to intensity and area involved; Intensity of inflammation was graded $0-3$ and the number of quadrants involved was recorded. A maximum score of 12 per eye was therefore possible.

Each patient was randomly allocated by the Pharmacy department to receive one of three treatments (labelled $\mathrm{A}, \mathrm{B}, \mathrm{C}$ ) to be instilled five times a day to the affected eye for three weeks. In patients presenting with bilateral disease, each eye was randomised separately. The three treatments were: gutt. prednisolone $0.3 \%$, gutt normal saline $0.5 \%$, or gutt flurbiprofen $0.03 \%$.

Each patient was reassessed as above on three occasions at weekly intervals. The treatment was continued for three weeks in every case even if the inflammation had resolved. The treatment was then tailed off over four days. Patients were asked to return in the event of a recurrence after treatment was discontinued.

\section{Results}

Sixty-three patients ( 77 eyes) with episcleritis were included in the study. Fourteen patients had bilateral disease either simultaneously (10 patients) or consecutively (4 patients).

There were 29 males and 34 females. The age range was 19 to 73 with an average of 39.4 years. Fifty-seven patients were of Caucasian origin, four were African and two were Asian.

Thirty-eight patients were presenting with their first episode whilst 25 patients had recurrent episcleritis. The duration of the history prior to presentation varied from one day to six weeks (mean $=9.3$ days).

Twenty-five eyes were treated with gutt. Flurbiprofen, 26 with gutt. normal saline and 26 with gutt. prednisolone.

Five patients on flurbiprofen and five patients from the placebo group were withdrawn from the trial. In six cases (two were using gutt. flurbiprofen and four gutt. placebo), this was because the inflammatory symptoms and signs had increased at a subsequent visit and had become compatible with a diagnosis of slceritis. One patient was withdrawn because he developed scleritis in his other eye, which necessitated oral treatment. One patient on flurbiprofen was withdrawn from the trial because she developed a follicular conjunctivitis (which promptly resolved on cessation of treatment) and another because the stinging sensation from instilling the drops was intolerable. One patient from the placebo group was withdrawn because she developed a rash on her arm which could have been an allergic reaction to her treatment.

None of the patients in the steroid-treated group were withdrawn from the trial.

\section{Analysis}

The mean scores for pain and episcleral injection are expressed graphically in Figures $1 \&$ 2.

The proportion of patients free of symptoms and signs was calculated for each group at weeks 1,2 and 3 . This is illustrated in Figures $3 \& 4$. These graphs show that episcleral injection and pain in the steroidtreated group resolved more rapidly than in either of the other two groups. Cure rates for each treatment group were compared statistically. The patients' results were stratified according to their initial score and analysed using the Mantel Haenszel test of statistical significance. The analysis compared each treatment at every visit.

Gutt prednisolone $0.3 \%$ is significantly better than gutt placebo and gutt flurbiprofen at curing pain (significant by two weeks $\mathrm{p}=0.04$ ) and episcleral injection (significant at three weeks $\mathrm{p}=0.015, \mathrm{p}=0.007$ ).

\section{Discussion}

The results of this double-blind trial show that gutt. flurbiprofen is no better than gutt. placebo at treating episcleritis.

This study confirms that in the majority of patients, resolution of symptoms and signs occurs rapidly, even with placebo. However, 


\section{SYMPTOMS}

AVERAGE SCORE

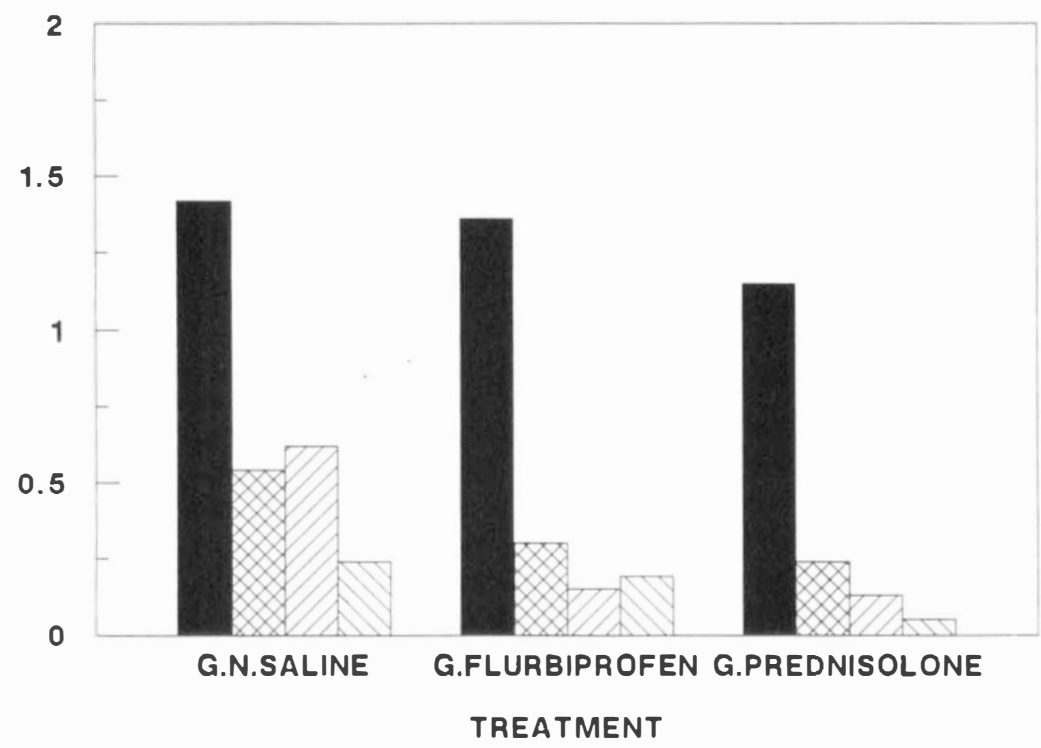

Fig. 1

\section{EPISCLERAL INJECTION}

\section{AVERAGE SCORE}

4

3

2

1

0

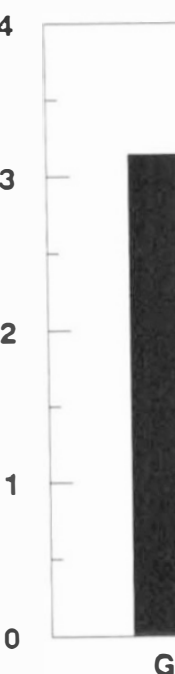

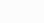


\% CURED

\section{SYMPTOMS : \% CURED}

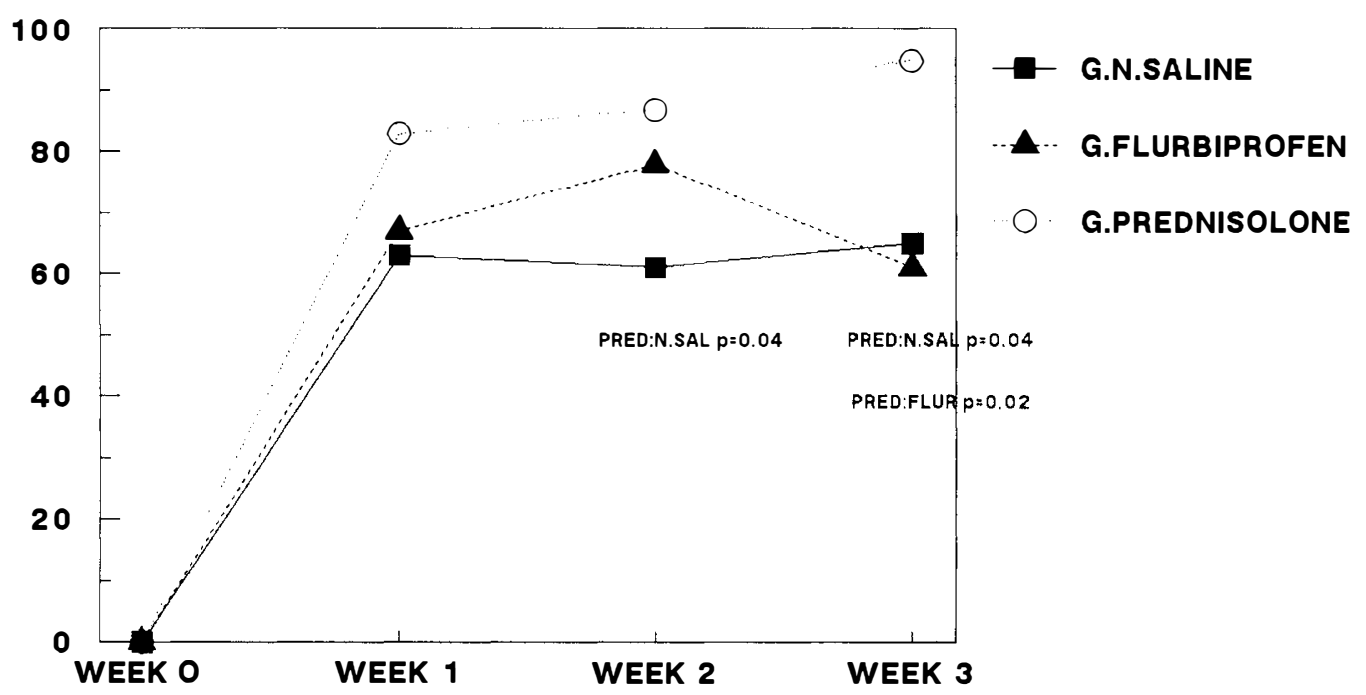

Fig. 3

EPISCLERAL INJECTION : \%CURED

\section{\% CURED}

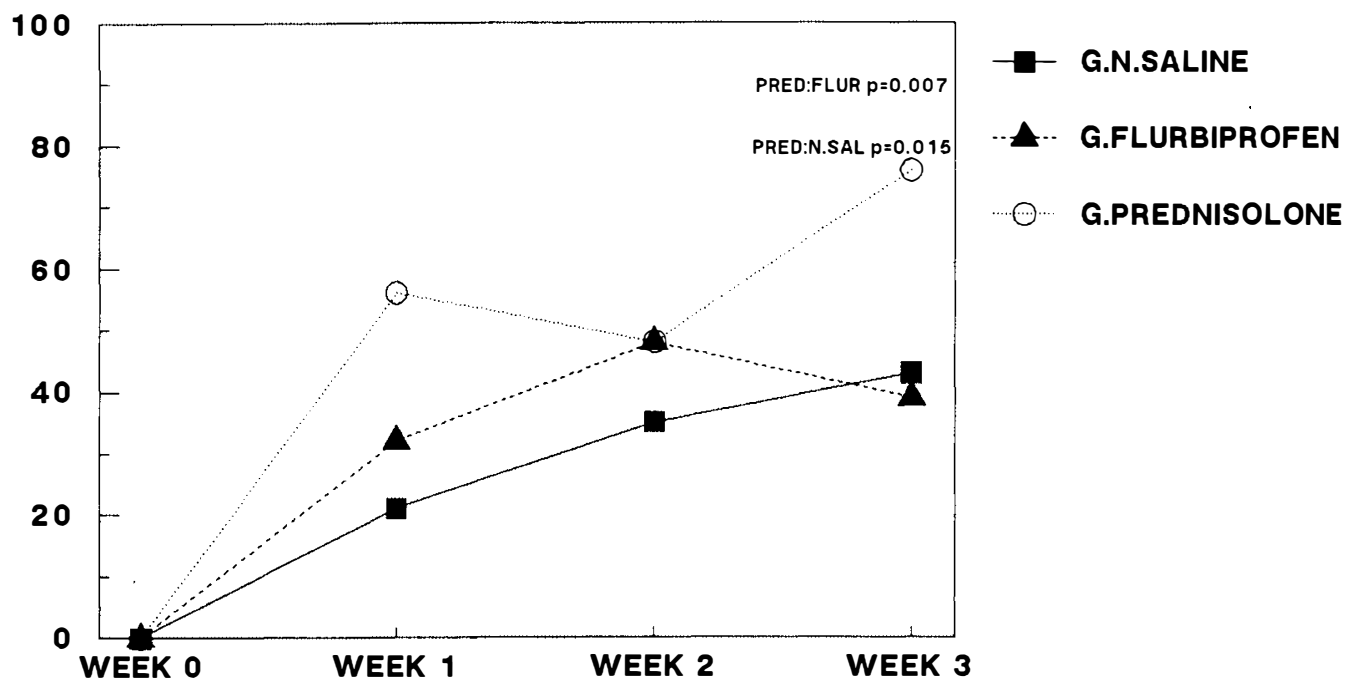

Fig. 4

Figs. 3 and 4. Proportion of patients free of symptoms and signs in each group at weeks $0,1,2$ and 3. 
the duration of the inflammation prior to many cases presenting to Casualty and the persistence of an inflammatory reaction even after three further weeks' treatment suggests that in some cases, episcleritis may not be self-limiting.

Clearly, the nature of the inflammatory process is very variable; six patients were withdrawn from the study due to the development of anterior scleritis in the affected eye. All of these responded well to a course of oral flurbiprofen $100 \mathrm{mg}$ tds. None of these patients were on topical steroids. It is possible that the progression of an inflammatory episode from episcleritis to scleritis can be aborted by the use of topical steroids. Further studies are required to elucidate this question.

Flurbiprofen sodium is a phenylalkanoic acid (2-(2-fluoro-4-biphenyl)-propionic acid. C15 H13 FO3.) which possesses anti-inflammatory, analgesic and antipyretic activity. It is thought to act by inhibition of prostaglandin synthetase. ${ }^{3}$ Although flurbiprofen is known to be effective in scleritis and episcleritis when administered orally, it does not appear to be a useful topical agent in the treatment of episcleritis. Carbon-14 studies $^{4}$ have shown the penetration of flurbiprofen into the cornea, sclera and aqueous to be good, being comparable to that of dipivefrin. The problem therefore is unlikely to be poor bioavailability.

As a prostaglandin synthetase inhibitor, flurbiprofen may be more useful in preventing the onset of inflammation than in settling it once initiated. However this does not explain the difference between the effectiveness of oral and topical preparations. Nine of the 25 patients treated with flurbiprofen described a stinging sensation on instillation. It is con- ceivable that as a result compliance was poorer in this group than in either of the other two.

\section{Conclusions}

This study confirms episcleritis to be a condition which is usually benign and self-limiting. However, the course of the inflammatory process can be variable and progression to scleritis was observed in six of 77 eyes.

Treatment with topical prednisolone has been shown to be superior to either topical flurbiprofen or placebo in achieving resolution of episcleral inflammation. Although no complications related to the use of topical prednisolone were encountered in this study, we would recommend that its use should be restricted to inflammation which has failed to improve spontaneously after one week.

The authors gratefully acknowledge the generous help of Allergan Pharmaceuticals in providing flurbiprofen drops for this study. We would also like to thank Dr D. Minassian for his invaluable help with the statistical analysis of the data and $\mathrm{Mr} \mathrm{V}$. Andrews and the staff of the pharmacy for their help with the preparation of the drops and randomisation of the patients.

\section{References}

${ }^{1}$ Watson PG, McKay DAR, Clemett RS, Wilkinson P: Treatment of episcleritis: A double-blind trial comparing betamethasone $0.1 \%$, Oxyphenbutazone $10 \%$, and placebo eye ointments. (1973) Brit J Ophthal 57: 866-70.

${ }^{2}$ Watson PG: Doyne Memorial Lecture 1982. Trans Ophthal Soc UK, 102: 257-81.

${ }^{3}$ Crook D, Collins AJ: Prostaglandin synthetase activity from human rheumatoid synovial tissues and its inhibition by non-steroidal anti-inflammatory drugs. Prostaglandins 1975, 9: 857.

${ }^{4}$ Anderson J, Shackleton M: Flurbiprofen absorption in ocular tissues. Report on file, Biochemistry dept, Allergan. 